\title{
ASYMMETRIES IN LONG-TERM AND SHORT-TERM PLASTICITY AT THALAMIC AND CORTICAL INPUTS TO THE AMYGDALA IN VIVO
}

\author{
Torfi Sigurdsson $^{1}$, Christopher K. Cain ${ }^{1}$, Valérie Doyère ${ }^{1,2}$, and Joseph E. LeDoux ${ }^{1}$ \\ ${ }^{1}$ Center for Neural Science, New York University, 4 Washington Place, New York, NY 10003, \\ USA; \\ ${ }^{2}$ NAMC, CNRS-UMR8620, Université Paris-Sud, 91405 Orsay, France
}

\begin{abstract}
Converging lines of evidence suggest that synaptic plasticity at auditory inputs to the lateral amygdala (LA) is critical for the formation and storage of auditory fear memories. Auditory information reaches the LA from both thalamic and cortical areas, raising the question whether they make distinct contributions to fear memory storage. Here we address this by comparing the induction of long-term potentation (LTP) at the two inputs in vivo in anesthetized rats. We first show, using field potential measurements, that different patterns and frequencies of highfrequency stimulation (HFS) consistently elicit stronger LTP at cortical inputs compared to thalamic inputs. Field potential responses elicited during HFS of thalamic inputs were also smaller compared to responses during HFS of cortical inputs, suggesting less effective postsynaptic depolarization. Pronounced differences in the short-term plasticity profiles of the two inputs were also observed: whereas cortical inputs displayed paired-pulse facilitation, thalamic inputs displayed paired-pulse depression. These differences in short-term and long-term plasticity were not due to stronger inhibition at thalamic inputs: although removal of inhibition enhanced responses to HFS, it did not enhance thalamic LTP and left paired-pulse depression unaffected. These results highlight the divergent nature of short-term and long-term plasticity at thalamic and cortical sensory inputs to the LA, pointing to their different roles in the fear learning system.
\end{abstract}

\section{Keywords \\ AMYGDALA; LTP; SHORT-TERM PLASTICITY; INHIBITION; EXTRACELLULAR RECORDINGS; FEAR}

\section{INTRODUCTION}

During fear conditioning, an initially neutral conditioned stimulus, typically a tone, acquires the ability to elicit fear responses after being paired with an aversive unconditioned stimulus, such as a mild footshock. Fear conditioning has emerged as a powerful tool for unraveling the neural mechanisms of learning and memory and may aid in the understanding and treatment of emotional disorders. A large body of evidence implicates the amygdala as a critical component of the circuitry underlying this form of learning (LeDoux, 2000). In particular, converging lines of evidence from pharmacological, electrophysiological and behavioral studies suggest that synaptic plasticity at auditory inputs to the lateral nucleus of

Correspondence should be sent to: Torfi Sigurdsson, Institute of Neurophysiology, Goethe University Frankfurt, Theodor-Stern-Kai 7 , 60590 Frankfurt, Germany, Phone: (49) 696301 84098, Fax: (49) 696301 6987, sigurdsson@em.uni-frankfurt.de. 
the amygdala (LA) plays a critical role in the formation and storage of auditory fear memories (Maren \& Quirk, 2004; Rodrigues et al., 2004; Sigurdsson et al., 2007).

Long-term potentiation (LTP) is a persistent form of synaptic plasticity that is widely studied as a cellular mechanism for learning and memory (Bliss \& Collingridge, 1993; Martin et al., 2000). LTP can be induced at sensory inputs to the LA both in vitro and in vivo (Weisskopf et al., 1999; Tsvetkov et al., 2002; Doyere et al., 2003; Humeau et al., 2005). Fear conditioning is also accompanied by LTP-like changes in synaptic transmission at auditory inputs to the LA (McKernan \& Shinnick-Gallagher, 1997; Rogan et al., 1997) and occludes artificially induced LTP (Tsvetkov et al., 2002; Schroeder \& ShinnickGallagher, 2005). Furthermore, pharmacological manipulations which block LTP in the amygdala also impair the acquisition and consolidation of conditioned fear (reviewed in Rodrigues et al., 2004). Collectively, these findings support the view that LTP-like changes in synaptic strength at sensory inputs to the LA underlie the formation and storage of fear memories (Sigurdsson et al., 2007).

The LA receives auditory information from both thalamic (Turner \& Herkenham, 1991; Bordi \& LeDoux, 1994; Doron \& Ledoux, 2000; Linke et al., 2000) and cortical (Mascagni et al., 1993; Romanski \& LeDoux, 1993; Shi \& Cassell, 1997; McDonald, 1998) areas. Although fear conditioning can be mediated via either the thalamic or cortical inputs (Romanski \& LeDoux, 1992), there is also evidence to suggest that they play distinct roles in the storage of fear memories (Campeau \& Davis, 1995; Armony et al., 1997; Boatman \& Kim, 2006). The nature of their individual contributions, however, remains poorly understood. Comparing synaptic plasticity at the two inputs can help shed light on this question. In the present study we therefore examined LTP induction as well as short-term plasticity at these inputs in vivo by directly stimulating the thalamic and cortical areas providing auditory input to the LA. We found that LTP was greater in magnitude at cortical compared to thalamic inputs across a range of induction protocols. Cortical inputs also displayed larger responses during high-frequency stimulation (HFS) as well as paired-pulse facilitation, whereas paired-pulse depression was observed at thalamic inputs. These results reveal highly divergent long-term and short-term plasticity at the thalamic and cortical inputs to the LA, pointing to their different roles in the fear learning system.

\section{MATERIALS AND METHODS}

\section{Subjects and surgery}

Experiments were performed using adult male Sprague-Dawley rats (360-430g) and were approved by NYU's University Animal Welfare Committee. Rats were anesthetized with Urethane (1.6-2.0 mg/kg, i.p.) and placed in a stereotaxic frame. The skull was exposed and three small craniotomies were made on the left side of the skull to allow the placement of a recording electrode in the lateral amygdala (LA; $3.0 \mathrm{~mm}$ posterior to bregma, $5.3-5.7 \mathrm{~mm}$ lateral to midline, $6.0-6.5 \mathrm{~mm}$ below dura) and stimulating electrodes in 1) the medial division of the medial geniculate and posterior intralaminar nuclei of the thalamus ( $\mathrm{MGm} /$ PIN; $5.0 \mathrm{~mm}$ posterior to bregma, $3.0 \mathrm{~mm}$ lateral to midline, $6.0 \mathrm{~mm}$ below dura) and 2) auditory association area TE3 $(5.8 \mathrm{~mm}$ posterior to bregma, $6.5-6.8 \mathrm{~mm}$ lateral to midline, $4.0 \mathrm{~mm}$ below dura). The final positions of electrodes were adjusted to maximize the responses to stimulation of either input.

\section{Stimulation and Recording of Neural Responses}

Neural activity was recorded in the LA using either a glass pipette filled with $0.9 \% \mathrm{NaCl}$ (impedance $=0.8-1.0 \mathrm{M} \Omega$ ) for recording field potentials or tungsten microelectrodes $(6-7$ $\mathrm{M} \Omega$; FHC, Bowdoinham, ME) for simultaneously recording single-unit responses. 
Stimulating electrodes were concentric bipolar electrodes with a $1 \mathrm{~mm}$ base-tip (anodecathode) separation (SNEX-100, Rhodes Medical Instruments).

For field potential recordings, the electrode signal was amplified (X1000), filtered (0.1 - 500 $\mathrm{Hz}$ ) and digitized at $10 \mathrm{kHz}$ using a CED Micro 1401 interface and Spike 2 software (Cambridge Electronic Design, Cambridge, UK). Input-output curves were derived by delivering single-pulse stimuli of increasing intensity $(100-800 \mu \mathrm{A}, 100 \mu \mathrm{s}$, in $100 \mu \mathrm{A}$ steps) to the MGm/PIN and TE3. The test stimulus intensities were then chosen so that stimulation of either input evoked responses of comparable magnitude in the LA and which were also $40-60 \%$ of the maximum response amplitude for each input.

In experiments involving single-unit recordings, the electrode signal was amplified X100010000 , filtered $(0.3-10 \mathrm{kHz})$ and digitized at $25 \mathrm{kHz}$. The electrode was placed just above the LA and then advanced in $10 \mu \mathrm{m}$ steps until action potentials (Signal-to-noise ratio > 3) could be reliably discerned in response to stimulation of either input. Only neurons which responded at a latency of $<15 \mathrm{~ms}$ were investigated. Test stimulation intensity was set so that a single spike was elicited on half of the trials. Parameters of the action potential waveforms were analyzed off-line to ensure single unit isolation and to determine whether spikes elicited by thalamic and cortical stimulation could be attributed to the same neuron.

\section{Paired-pulse stimulation}

Paired-pulse stimulation was used to examine the presynaptic properties of the thalamic and cortical inputs to the LA (Zucker \& Regehr, 2002). Paired stimuli were delivered to the MGm/PIN and TE3 in an interleaved fashion, with each region stimulated every 20s. Interstimulus intervals of 25,50,100,200,600 and 1000ms were tested in an ascending order, and each interval was tested three times. However, because paired-pulse responses at the shortest ISI were highly variable between batches of animals and were also strongly influenced by the ongoing response to the first stimulus, this ISI was excluded from the final analysis. The exclusion of this ISI did not, however, change the main results. For field potentials the paired-pulse ratio (PPR) was calculated by expressing the amplitude of the response to the second stimulus in a pair as the percentage of the response to the first stimulus. The stimulus intensity was set so that the first stimulus evoked a response that was $40-60 \%$ of the maximum. For single-unit responses, the PPR was the probability of evoking a spike following the second stimulus, expressed as the percentage of the probability of evoking a spike following the first stimulus in a pair. Stimulation intensity was set so that the probability of evoking a spike following the first stimulus was 50\%. Because this methodology places an upper limit on the amount of facilitation that can be observed in single-unit responses (at most 200\%) but not on depression, facilitation may have been underestimated for some of the neurons studied.

\section{Long-Term Plasticity}

Long-term plasticity at thalamic and cortical inputs was examined by inducing long-term potentiation (LTP). First, a baseline was established by delivering test stimuli to MGm/PIN and TE3 in an interleaved fashion once per minute for 20 mins. LTP was then induced in one pathway using five tetanic trains (100 stimuli at 25,100 or $200 \mathrm{~Hz}$ ) delivered at a 3 minute inter-train interval. Following LTP induction, test stimulation resumed for an additional 60 minutes. For analysis of LTP, the response amplitudes were expressed as a percentage of the average response amplitude during the baseline period prior to LTP induction. We also included in the analysis the results from a separate unpublished study in which LTP was induced using theta-burst stimulation (TBS), consisting of 3 series of 10 trains $(100 \mathrm{~Hz}, 100 \mathrm{~ms}$ duration, $200 \mathrm{~ms}$ inter-train interval), delivered once a minute (Doyere et al., 2003). In this experiment, animals received an intraperitoneal injection of $1 \mathrm{ml}$ saline 
vehicle 1 hour prior to the experiment which our unpublished observations suggest does not have any discernible effect on LTP.

\section{Pharmacological manipulations}

In a subset of experiments, the effects of inhibition on synaptic transmission and plasticity were tested using the $\mathrm{GABA}_{\mathrm{A}}$ receptor antagonist picrotoxin and the $\mathrm{GABA}_{\mathrm{B}}$ receptor antagonists CPG35348 (Sigma, St. Louis, MO). The drugs were added to the saline solution of the recording pipette, yielding a final concentration of $5 \mathrm{mM}$ for picrotoxin and $40 \mathrm{mM}$ for CPG35348. The drugs were allowed to diffuse passively into the extracellular space and their effects were assessed by comparing evoked responses to those recorded in control animals with only saline in the pipette.

\section{Histology}

At the end of experiments where glass pipettes were used for recording, a solution containing 4\% Pontamine Skye Blue was injected into the pipette and then ejected iontophoretically ( $20 \mu \mathrm{A}, 5 \mathrm{mins})$. In experiments using tungsten microelectrodes, a small lesion $(80 \mu \mathrm{A}, 3 \mathrm{~s})$ was made at several dorsoventral locations to help reconstruct the electrode track. Animals were then decapitated and the brains removed and stored in a buffered formalin solution containing 30\% sucrose. Brains were sectioned at $50 \mu \mathrm{m}$ and examined under light microscopy to verify placement of recording and stimulating electrodes.

\section{RESULTS}

\section{Electrode placements and basic response properties}

Placements of stimulating and recording electrodes are shown in Figure 1. In the thalamus, the electrodes targeted primarily the medial PIN and the dorsally adjacent MGm. In the cortex, area TE3 was primarily stimulated, although in some animals the electrode tips extended into the ventrally adjacent perirhinal cortex, which is also a potential source of auditory inputs to the LA (McDonald, 1998; Lindquist et al., 2004). Recording sites were exclusively located in the dorsal portion of the LA.

Stimulation of either the MGm/PIN or TE3 (from now on referred to as the thalamic and cortical inputs, respectively), elicited a field potential response in the LA consisting of an early negative response and a later positive response (Figure $2 A$, upper traces). The trough of the negative response typically coincided with evoked unit activity (see bottom traces in Figure $2 A$ ), and we also found that it is enhanced by blocking $\mathrm{GABA}_{\mathrm{A}}$ receptors (see Figure 6 below). This suggests that the negative response reflects the net excitatory synaptic input onto LA neurons,(Mitzdorf, 1985; Leung, 1990). The latency of the negative response is consistent with a monosynaptic origin, as suggested by antidromic response latencies in thalamus and cortex following electrical stimulation of LA (T. Sigurdsson, unpublished observations). The amplitude of the negative response increased with increasing stimulus intensities (Figure $2 B$ ), and the input-output curves did not differ between the thalamic and cortical inputs. In subsequent experiments, the amplitude of the negative response was chosen for analysis and stimulation intensities were set so that evoked response amplitudes were $40-60 \%$ of the maximum.

\section{Asymmetric induction of long-term potentiation at thalamic and cortical inputs to the lateral amygdala}

In a previous study using freely moving rats we demonstrated that LTP at cortical inputs is greater in magnitude compared to thalamic inputs following theta burst stimulation (Doyere et al., 2003). The first goal of the present study was therefore to examine whether this 
asymmetry in LTP was specific to particular induction protocols. To test this, we first induced LTP using a standard protocol consisting of five trains of high-frequency stimulation (HFS; $100 \mathrm{~Hz} \times 1$ s, 3 minute inter-train interval; Huang \& Kandel, 1998). In each experiment, one input received HFS whereas the other served as a control pathway for possible heterosynaptic effects. As shown in Figures $3 A$, this protocol induced modest but significant LTP at the thalamic inputs, measured 55-60 minutes after induction (112 $\pm 1.3 \%$, $\mathrm{p}<0.01)$, whereas the same protocol induced much larger LTP at cortical inputs $(167 \pm 20 \%$, $\mathrm{p}<0.05$ ). We next examined the frequency-dependence of this difference in separate groups of animals by delivering HFS trains at different frequencies $(25$ and $200 \mathrm{~Hz}$ ) while keeping the number of stimuli constant (100 stimuli/train in all experiments). The results of these experiments are summarized in Figure $3 B$. At all frequencies LTP appeared larger at cortical compared to thalamic inputs. This was confirmed by a two-way ANOVA (input by frequency) which revealed a main effect of input $\left(\mathrm{F}_{1,18}=14.14, \mathrm{p}<0.01\right)$, without a significant interaction $\left(\mathrm{F}_{2,18}=0.89, \mathrm{p}=0.43\right)$. No heterosynaptic effects of HFS were observed at either input at any frequency ( $>0.05$, data not shown). In an additional group of animals, we induced LTP with the theta burst protocol used in our previous study (Doyere et al., 2003), which has been suggested to mimic naturally occurring patterns of activity in the brain (Otto et al. 1991). Consistent with our previous results, this induced significantly larger LTP at cortical compared to thalamic inputs (Figure 3C; $133 \pm 6.1 \%$ vs. $110 \pm 3.1 \%$, $\mathrm{p}<0.05)$. Taken together, these results suggest that LTP is more robust at cortical compared to thalamic inputs to the LA following HFS, and this difference does not appear to depend on a particular stimulation frequency or induction protocol.

\section{Responses to high-frequency stimulation are larger at cortical inputs}

We next set out to investigate possible mechanisms underlying the differences in LTP between the two inputs. Because postsynaptic depolarization is a critical determinant of LTP magnitude (Gustafsson et al., 1987; Bliss \& Collingridge, 1993), it is possible that HFS of thalamic inputs is less effective at depolarizing LA neurons than HFS of cortical inputs. To examine this possibility, we analyzed the field potential responses during HFS. As shown in Figure $4 A$, HFS elicited a sustained negative field potential in the LA, which may reflect the summation of excitatory postsynaptic potentials in LA neurons (Wigstrom \& Gustafsson, 1984; Grover \& Teyler, 1990). To analyze these responses further, the raw voltage traces were digitally filtered to remove stimulus artifacts (Figure $4 A$ ) and averaged across animals. Figure $4 B$ shows the group averaged field potential response during HFS at $100 \mathrm{~Hz}$. At both inputs, HFS caused a sustained negative field potential response which was considerably larger at cortical inputs. We further quantified these responses by computing the mean field potential during HFS and compared this value between inputs and across frequencies. As can be seen from Figure 4C, increasing the HFS frequency increased the responses during the trains and these were larger at cortical compared to thalamic inputs. We confirmed this with a two-way ANOVA (input by frequency), which revealed a main effect of input $\left(\mathrm{F}_{1,18}=60.26, \mathrm{p}<0.01\right)$, and frequency $\left(\mathrm{F}_{2,18}=21.97, \mathrm{p}<0.01\right)$ as well as a significant interaction $\left(\mathrm{F}_{2,18}=10.20, \mathrm{p}<0.01\right)$. Subsequent post-hoc tests (Bonferroni's test) revealed a difference between the two inputs at $100 \mathrm{~Hz}$ and $200 \mathrm{~Hz}(\mathrm{p}<0.01)$, but not at $25 \mathrm{~Hz}(\mathrm{p}>0.05)$.

One possible explanation for these findings is that individual cortical stimuli were more effective at driving LA cells than thalamic stimuli, thus producing larger overall HFS responses. However, this is unlikely since single test pulses delivered to either input prior to HFS (at $0.017 \mathrm{~Hz}$ ) evoked field potential responses of the same magnitude in the LA (Figure $4 D$; thalamic: $0.56 \pm 0.04 \mathrm{mV}$; cortical: $0.64 \pm 0.05 \mathrm{mV}$; $\mathrm{p}>0.05$ ). These results therefore suggest that the thalamic and cortical inputs differ specifically in their responsiveness to HFS, and this in turn may explain their differential susceptibility to LTP induction. 


\section{Thalamic and cortical inputs display different short-term plasticity profiles}

During high-frequency stimulation, synapses can undergo short-term changes in their efficacy that powerfully modulate how they convert presynaptic activity into postsynaptic responses (Abbott \& Regehr, 2004). For example, when two presynaptic stimuli are delivered in close succession, the amplitude of the response to the second stimulus is often larger (facilitation) or smaller (depression) than the response to the first, depending on a number of factors including the type of synapse being studied (Zucker \& Regehr, 2002). We therefore examined whether the different responses to HFS at the thalamic and cortical inputs were associated with differences in short-term plasticity at the two inputs. To this end, we delivered interleaved pairs of test stimuli to the auditory thalamus or cortex and examined paired-pulse responses at inter-stimulus intervals (ISIs) between 50 and $1000 \mathrm{~ms}$. We quantified the paired-pulse response by expressing the response to the second stimulus as a $\%$ of the response to the first. Figure $5 A, B$ shows the average paired-pulse response of the two inputs as a function of ISI. At thalamic inputs paired-pulse depression was observed at all ISIs ( $\mathrm{p}<0.01 \mathrm{vs.}$ the $1^{\text {st }}$ response at all ISIs, Bonferroni's t-test). In contrast, pairedpulse stimulation of the cortical inputs revealed facilitation at ISIs between 50 and $200 \mathrm{~ms}$ ( $\mathrm{p}<0.01$, Bonferroni's t-test). This difference between the inputs was confirmed with a twoway ANOVA (input by ISI), which revealed a main effect of input $\left(\mathrm{F}_{4,55}=5.31, \mathrm{p}<0.01\right)$ and significant differences at 50 to $500 \mathrm{~ms}$ ISIs (Bonferroni t-test, $\mathrm{p}<0.001$ ).

Because field potentials reflect the summed activity of a large number of neurons, one possible explanation for these results is that the different paired-pulse ratios reflect differences in the neuronal populations that are targeted by the thalamic and cortical inputs rather than differences in the inputs per se. To address this, we recorded paired-pulse responses of well-isolated single neurons responding to stimulation of both thalamic and cortical inputs. A total of 25 neurons were recorded, of which 8 responded exclusively to thalamic stimulation, 8 exclusively to cortical stimulation and 9 were responsive to stimulation of both structures. In the absence of stimulation, the majority of neurons (22/25) were either silent or had very low levels of spontaneous activity $(<1 \mathrm{~Hz})$, suggesting that we primarily sampled projection neurons (Likhtik et al., 2006). Evoked responses typically consisted of a single spike at a short latency $(<10 \mathrm{~ms})$ and stimulus intensities were set so that the first pulse in a pair elicited a response on average $50 \%$ of the time.

The results of these experiments confirmed those previously obtained using field potential measurements. For the subset of neurons responding to both thalamic and cortical stimulation ( $\mathrm{n}=9$ ), we quantified the paired-pulse response by expressing the probability of eliciting a spike following the second stimulus as a percentage of the probability of eliciting a spike following the first stimulus (Figure $5 C, D$ ). This revealed clear differences in the paired-pulse responses of the two inputs, confirmed by a main effect of input in a two-way ANOVA $\left(\mathrm{F}_{1,40}=24.39, \mathrm{p}<0.01\right)$. Analysis of all cells $(\mathrm{n}=17$ for each input) confirmed this effect ( $\mathrm{p}<0.01$, data not shown) and revealed consistent paired-pulse depression in response to thalamic stimulation at every ISI between 50 and $1000 \mathrm{~ms}$ (Bonferroni t-tests; $\mathrm{p}<0.01$ ). In contrast, paired-pulse responses to cortical stimulation were more heterogeneous and on average neither facilitation nor depression was observed at any ISI (Bonferroni t-tests; $\mathrm{p}>0.05$ ). The lack of facilitation at cortical inputs differs from the results we obtained by measuring field potential responses for reasons that are not clear. Nevertheless, both the single-unit and field potential results are in qualitative agreement with each other in revealing marked asymmetries in the paired-pulse response profiles of the thalamic and cortical inputs. 


\section{Weaker LTP at thalamic inputs is not attributable to stronger inhibition}

Inhibitory transmission can powerfully constrain excitatory responses (Pouille and Scanziani 2001) and changes in the balance between excitatory and inhibitory transmission can contribute to short-term plasticity of the kind described in the previous section (Nathan \& Lambert, 1991; Li et al., 1996; Marder \& Buonomano, 2003). Inhibitory transmission can also modulate responses to HFS (Wigstrom and Gustafsson 1984) and impair, or even prevent, the induction of LTP (Wigstrom \& Gustafsson, 1983b; a; Bissiere et al., 2003). We therefore examined whether the strong paired-pulse depression, weaker HFS responses and smaller LTP at thalamic inputs was due to stronger inhibition at these inputs compared to the cortical inputs. According to this hypothesis, pharmacological blockade of inhibitory transmission should facilitate LTP at thalamic inputs, making it more comparable in magnitude to LTP at cortical inputs.

To test this, we repeated the above experiments with picrotoxin, an antagonist of $\mathrm{GABA}_{\mathrm{A}}$ receptors, in the recording pipette solution and allowed the drug to diffuse passively into the extracellular space (see Materials and Methods). Responses recorded with picrotoxin in the pipette were considerably larger than those recorded with saline alone, both in terms of amplitude and response duration (Figure $6 A, B$ ). We therefore quantified the effect of picrotoxin by calculating the area of the negative component of the field potential response (see inset in Figure 6C) at different stimulation intensities and compared the input-output relationship to that recorded with only saline in the pipette. As Figure $6 C, D$ shows, picrotoxin greatly enhanced the evoked responses at both thalamic and cortical inputs. Comparing the mean response area (by averaging across stimulation intensities) revealed significant effects of picrotoxin at both thalamic (saline: $29 \pm 2.3 \mathrm{mV}$; picrotoxin: $137 \pm$ $12.5 \mathrm{mV}$; $\mathrm{p}<0.05$; Bonferroni's t-test) and cortical inputs (saline: $37 \pm 4.9 \mathrm{mV}$; picrotoxin: $188 \pm 30.4 \mathrm{mV} ; \mathrm{p}<0.05$ ). To compare the magnitude of this effect between the two inputs, we normalized the mean responses recorded with picrotoxin (averaged across stimulation intensities) by expressing them as a percentage of the mean responses recorded with saline. This revealed comparable levels of enhancement for the two inputs (thalamic: $455 \pm 41.4 \%$; cortical: $483 \pm 78.4 \%$; $>0.05$ ). These results suggest that under normal circumstances inhibitory transmission constrains the amplitude and duration of excitatory responses, and that it does so to a comparable extent at the thalamic and cortical inputs.

We next examined whether inhibitory transmission contributes to the different short-term plasticity profiles of the thalamic and cortical inputs by examining responses to paired-pulse stimulation in the presence of picrotoxin. As shown in Figure $7 A, C$, paired-pulse responses at the thalamic inputs were unaffected by picrotoxin, which was confirmed with a two-way ANOVA (drug by ISI) that failed to show a significant effect of drug $\left(\mathrm{F}_{1,88}=1.61, \mathrm{p}>0.05\right)$. Although there was a significant drug by ISI interaction $\left(\mathrm{F}_{4,88}=2.73, \mathrm{p}>0.05\right)$, post-hoc tests failed to reveal a drug effect at any of the ISIs (Bonferroni's t-test; $p>0.05$ ). At cortical inputs, in contrast, paired-pulse facilitation was abolished by picrotoxin (Figure $7 B, D$ ), as revealed by a main effect of drug $\left(\mathrm{F}_{1,88}=19.88, \mathrm{p}<0.01\right)$, a significant drug by ISI interaction $\left(\mathrm{F}_{4,88}=10.82, \mathrm{p}<0.01\right)$ and significant drug effects at ISIs between 50 and $200 \mathrm{~ms}$ (Bonferroni's t-test; $\mathrm{p}<0.05$ ). These results suggest that under normal circumstances facilitation at cortical inputs may result in part from disinhibition (Nathan \& Lambert, 1991; see discussion; Li et al., 1996; Marder \& Buonomano, 2003). Importantly, however, the differences in the paired-pulse response profiles of the two inputs remained significant even in the presence of picrotoxin. A two-way ANOVA (input by ISI) on paired-pulse responses in the presence of picrotoxin revealed a main effect of input $\left(\mathrm{F}_{1,88}=10.00, \mathrm{p}<0.01\right)$, as well as an input by ISI interaction $\left(\mathrm{F}_{4,88}=2.59, \mathrm{p}<0.05\right)$ and a significant difference at the $50 \mathrm{~ms}$ ISI (Bonferroni's t-test, $\mathrm{p}<.05$ ). We also examined the contribution of $\mathrm{GABA}_{\mathrm{B}}$ receptors to paired-pulse responses by including the $\mathrm{GABA}_{\mathrm{B}}$ antagonist $\mathrm{CPG} 35348$ in the recording pipette in a seperate group of animals $(n=3)$. No effects of the drug were discernible at either 
input (data not shown). Thus, differences in inhibitory transmission cannot account entirely for the divergent short-term plasticity profiles of the thalamic and cortical inputs to the LA.

Finally, we examined whether blockade of inhibition would enhance responses to HFS and facilitate LTP at thalamic inputs, thus making it more comparable to the LTP observed at cortical inputs. As can be seen in Figure 8A, picrotoxin enhanced responses to $100 \mathrm{~Hz}$ HFS at thalamic inputs. This was confirmed with a two-way ANOVA (drug by time) which revealed a main effect of drug $\left(\mathrm{F}_{1,2786}=13.26, \mathrm{p}<0.05\right)$. However, despite the enhanced HFS responses, LTP at thalamic inputs was not facilitated in the presence of picrotoxin (Figure $8 C$, saline: $112 \pm 1.3 \%$; picrotoxin: $106 \pm 2.4 \%$; $>0.05$ ). In contrast to thalamic inputs, picrotoxin did not enhance responses to HFS of cortical inputs (Figure $8 B$ ), as shown by a lack of a drug effect in a drug by time ANOVA $\left(\mathrm{F}_{1,2388}=0.59, \mathrm{p}<0.05\right)$ and the magnitude of LTP at cortical inputs was not significantly different from that observed with saline (Figure $8 D ; \mathrm{p}>0.05)$. Importantly, however, in the presence of picrotoxin LTP remained significantly larger at cortical compared to thalamic inputs $(\mathrm{p}<0.05)$. Similar results were obtained in the presence of CPG 35348 (thalamic LTP: $115 \pm 10.5 \%$; cortical LTP: $151 \pm$ $12.1 \%$; data not shown). These results therefore argue against the hypothesis that differences in inhibitory transmission are responsible for the differences in LTP magnitude between the thalamic and cortical inputs to the LA in vivo.

\section{DISCUSSION}

\section{Asymmetric LTP induction at thalamic and cortical inputs to the LA in vivo}

Although considerable evidence suggests that long-term plasticity at auditory inputs to the LA underlies the formation and storage of auditory fear memories (Sigurdsson et al., 2007) one outstanding question is whether the thalamic and cortical auditory inputs to the LA play different roles in this process. This motivated us to ask, in the present study, how and why the induction of LTP differs at the two inputs. We previously showed that LTP is more readily induced at cortical compared to thalamic inputs to the LA following theta-burst stimulation in freely behaving rats (Doyere et al., 2003). Here we have extended these findings in the anesthetized rat by showing that this difference is observed across a range of induction protocols and frequencies.

While these results do not necessarily imply that thalamic inputs are less capable of synaptic change than cortical inputs, they at least suggest that plasticity at these two inputs may be sensitive to different patterns of pre- and postsynaptic activity. Supporting this interpretation, in vitro studies have shown that some stimulation protocols induce LTP only at cortical inputs (Humeau et al., 2003; Shin et al., 2006; Huang \& Kandel, 2007) whereas others favor LTP at thalamic inputs (Humeau et al., 2005). Interestingly, another in vivo study targeting the perirhinal cortical inputs to LA (Yaniv et al., 2000) found them less susceptible to LTP induction than thalamic inputs, suggesting that LTP may differ even within the cortical regions that provide auditory inputs to the LA. Although the functional significance of these differences is unclear, they may enable plasticity at these two inputs to the LA to encode different aspects of emotional memories.

\section{Differences in HFS responses and short-term plasticity profiles at thalamic and cortical inputs}

To begin to understand why LTP is differentially induced at thalamic and cortical inputs, we examined how they respond during high-frequency stimulation (HFS). We found that HFS of either input caused a sustained negative field potential response, which may reflect the summation of excitatory postsynaptic potentials (Wigstrom \& Gustafsson, 1984; Grover \& Teyler, 1990). However, HFS of cortical inputs evoked considerably larger responses in the 
LA than did HFS of thalamic inputs. This suggests that cortical inputs were more effective at depolarizing LA neurons, which could help explain the relatively more robust LTP at these inputs. Importantly, the larger responses elicited by HFS of cortical inputs were not due to individual cortical stimuli being more effective per se, since the responses to individual test pulses did not differ between the two inputs. This suggests that the two inputs display different dynamics during repeated stimulation that make them differentially responsive to HFS.

To examine this more closely, we compared the short-term plasticity profiles of the two inputs using paired-pulse stimulation (Zucker \& Regehr, 2002; Abbott \& Regehr, 2004). This revealed dramatic differences between the two inputs. In response to cortical stimulation, field potential responses displayed paired-pulse facilitation whereas pairedpulse depression was observed in response to thalamic stimulation. Differences in pairedpulse responses were also evident in responses of single neurons that were responsive to both thalamic and cortical stimulation, unambiguously demonstrating the differences between the two inputs. Although ours is the first study to systematically compare pairedpulse responses at the thalamic and cortical inputs in vivo, our results are in general agreement with previous studies that have examined the two inputs individually (Rosenkranz \& Grace, 2001; Zinebi et al., 2001; Zinebi et al., 2002; Sokal et al., 2005).

\section{Differences in inhibitory transmission do not account for asymmetries in LTP induction at the thalamic and cortical inputs}

The differences in short-term plasticity, HFS responses and LTP magnitude between the two inputs could reflect stronger feedforward inhibition at thalamic inputs ( $\mathrm{Li}$ et al., 1996; Shin et al., 2006). To examine this possibility, we repeated our experiments with picrotoxin, an antagonist of $\mathrm{GABA}_{\mathrm{A}}$ receptors, applied locally at the recording site. Picrotoxin increased the amplitude and duration of evoked field potential responses, suggesting that inhibition constrains excitatory responses in the LA under normal circumstances. However, the magnitude of this effect was comparable for both inputs, arguing against the hypothesis that inhibition is stronger at thalamic inputs. Furthermore, picrotoxin did not affect paired-pulse depression at thalamic inputs, suggesting that inhibitory transmission does not underlie this phenomenon. Interestingly, however, picrotoxin reduced paired-pulse facilitation at cortical inputs, suggesting that facilitation may be partly mediated by disinhibition (Nathan \& Lambert, 1991; Marder \& Buonomano, 2003), for example through paired-pulse depression at inhibitory inputs (Szinyei et al., 2000). Finally, we examined the effects of picrotoxin on HFS responses and LTP magnitude. Although picrotoxin enhanced responses to HFS at thalamic inputs it surprisingly did not increase the magnitude of LTP, which remained much smaller than at cortical inputs. This suggests that under normal circumstances the magnitude of LTP at thalamic inputs is not simply due to their relatively weak HFS responses. The $\mathrm{GABA}_{\mathrm{B}}$ receptor antagonist CPG35348,, did also not have any discernible effect on pairedpulse responses, responses to HFS or LTP magnitude at either input. These results lead us to conclude that differences in inhibitory transmission do not account for the asymmetries in LTP or short-term plasticity at the thalamic and cortical inputs in vivo.

In contrast to our findings, in vitro studies have found that removal of inhibition facilitates LTP at thalamic inputs to the LA (Bissiere et al., 2003) and eliminates differences in LTP between thalamic and cortical inputs (Shin et al., 2006; but see Humeau et al. 2005). Several possibilities may account for the discrepancy between these findings and ours. First, the in vitro studies used stimulation protocols which induced LTP only when inhibitory transmission was blocked whereas our stimulation protocols induced LTP with inhibition present. It is therefore possible that picrotoxin might have enhanced LTP in our experiments had we used stimulation protocols that were subthreshold for LTP induction under normal conditions. Another possibility is that the induction protocols used in vitro, in which 
presynaptic stimulation was paired with postsynaptic action potentials, may be more sensitive to inhibition than the HFS protocols we used. Finally, it is important to note that in our study the two auditory inputs were studied by directly stimulating their sources in the thalamus and cortex, whereas in vitro they are typically activated by stimulating the internal and external capsules. Although the two capsules carry fibers from auditory thalamus and cortex, they also have fibers from other thalamic and cortical areas. This might lead to some differences between results obtained in vivo and in vitro.

The mechanisms underlying the asymmetries in LTP between the thalamic and cortical inputs to the LA in vivo remain unclear, although our findings argue against differences in inhibition being responsible. One possibility is that the large asymmetries in LTP between the two inputs are causally related to the pronounced differences in their short-term plasticity profiles. Indeed, for many synapses the type of short-term plasticity that they display is correlated with their susceptibility to LTP induction. Specifically, facilitating synapses have been found to be capable of greater LTP than depressing synapses (Bolshakov \& Siegelbaum, 1995; Son \& Carpenter, 1996; Kleschevnikov et al., 1997; Volgushev et al., 1997; Commins et al., 1998; Akopian et al., 2000; Hardingham et al., 2007), which is consistent with the differences we observed between the thalamic and cortical inputs. This may be due to lower presynaptic release probability at facilitating synapses, which therefore have greater scope for LTP via presynaptic changes. Indeed, artificially increasing or decreasing release probability has been shown to impair or facilitate LTP induction, respectively, in the hippocampus (Bolshakov and Siegelbaum 1995; Schulz et al. 1997). Whether these or other mechanisms account for the asymmetries in LTP at thalamic and cortical inputs to the LA remains to be determined.

\section{Functional consequences}

The pronounced asymmetries in short-term and long-term plasticity at the thalamic and cortical inputs to the LA are likely to reflect their different contributions to fear learning. The greater susceptibility of cortical inputs to LTP induction may suggest a more prominent role in long-term storage of fear memories, as has been suggested by lesion studies (Campeau \& Davis, 1995; Boatman \& Kim, 2006). Alternatively, as suggested above, LTP at the two inputs may be sensitive to different patterns of pre- and postsynaptic activity, and therefore store different aspects of fear memories. The differences in short-term plasticity are also likely to affect the manner in which sensory information is transmitted to the LA (Abbott \& Regehr, 2004). It has been suggested that thalamic inputs carry a fast but crude auditory representation to the LA whereas cortical inputs relay information more slowly but with greater detail (Armony et al., 1997). Cortical inputs, which respond more effectively during sustained presynaptic stimulation, may be more suitable for transmitting complex auditory stimuli whose properties unfold over time, such as animal vocalizations (Lindquist et al., 2004). In contrast, the strongly depressing thalamic inputs may act primarily to signal the onset or novelty of a stimulus. Indeed, habituation is a prominent feature of the auditory responses of LA neurons (Bordi \& LeDoux, 1992; Herry et al., 2007), possibly mediated via-pulse depression. at thalamic inputs. Further elucidating the functional roles of synaptic plasticity at the thalamic and cortical inputs to the LA will be important in future studies.

\section{Acknowledgments}

The research was supported by grants R01 MH46516, R37 MH38774, K05 MH067048, P50 MH58911 to J.E L; CNRS-NSF Grant 17089 to V.D. and J.E.L; CNRS-PICS to VD; and an NRSA to CKC (F32MH077458) from the NIMH. 


\section{References}

Abbott LF, Regehr WG. Synaptic computation. Nature. 2004; 431:796-803. [PubMed: 15483601]

Akopian G, Musleh W, Smith R, Walsh JP. Functional state of corticostriatal synapses determines their expression of short- and long-term plasticity. Synapse. 2000; 38:271-280. [PubMed: 11020230]

Armony JL, Servan-Schreiber D, Romanski LM, Cohen JD, LeDoux JE. Stimulus generalization of fear responses: effects of auditory cortex lesions in a computational model and in rats. Cereb Cortex. 1997; 7:157-165. [PubMed: 9087823]

Bissiere S, Humeau Y, Luthi A. Dopamine gates LTP induction in lateral amygdala by suppressing feedforward inhibition. Nat Neurosci. 2003; 6:587-592. [PubMed: 12740581]

Bliss TV, Collingridge GL. A synaptic model of memory: long-term potentiation in the hippocampus. Nature. 1993; 361:31-39. [PubMed: 8421494]

Boatman JA, Kim JJ. A thalamo-cortico-amygdala pathway mediates auditory fear conditioning in the intact brain. Eur J Neurosci. 2006; 24:894-900. [PubMed: 16930417]

Bolshakov VY, Siegelbaum SA. Regulation of hippocampal transmitter release during development and long-term potentiation. Science. 1995; 269:1730-1734. [PubMed: 7569903]

Bordi F, LeDoux J. Sensory tuning beyond the sensory system: an initial analysis of auditory response properties of neurons in the lateral amygdaloid nucleus and overlying areas of the striatum. $\mathrm{J}$ Neurosci. 1992; 12:2493-2503. [PubMed: 1613543]

Bordi F, LeDoux JE. Response properties of single units in areas of rat auditory thalamus that project to the amygdala. I. Acoustic discharge patterns and frequency receptive fields. Exp Brain Res. 1994; 98:261-274. [PubMed: 8050512]

Campeau S, Davis M. Involvement of subcortical and cortical afferents to the lateral nucleus of the amygdala in fear conditioning measured with fear-potentiated startle in rats trained concurrently with auditory and visual conditioned stimuli. J Neurosci. 1995; 15:2312-2327. [PubMed: 7891169]

Commins S, Gigg J, Anderson M, O’Mara SM. Interaction between paired-pulse facilitation and longterm potentiation in the projection from hippocampal area CA1 to the subiculum. Neuroreport. 1998; 9:4109-4113. [PubMed: 9926856]

Doron NN, Ledoux JE. Cells in the posterior thalamus project to both amygdala and temporal cortex: a quantitative retrograde double-labeling study in the rat. J Comp Neurol. 2000; 425:257-274. [PubMed: 10954844]

Doyere V, Schafe GE, Sigurdsson T, LeDoux JE. Long-term potentiation in freely moving rats reveals asymmetries in thalamic and cortical inputs to the lateral amygdala. Eur J Neurosci. 2003; 17:2703-2715. [PubMed: 12823477]

Grover LM, Teyler TJ. Two components of long-term potentiation induced by different patterns of afferent activation. Nature. 1990; 347:477-479. [PubMed: 1977084]

Gustafsson B, Wigstrom H, Abraham WC, Huang YY. Long-term potentiation in the hippocampus using depolarizing current pulses as the conditioning stimulus to single volley synaptic potentials. J Neurosci. 1987; 7:774-780. [PubMed: 2881989]

Hardingham NR, Hardingham GE, Fox KD, Jack JJ. Presynaptic efficacy directs normalization of synaptic strength in layer 2/3 rat neocortex after paired activity. J Neurophysiol. 2007; 97:29652975. [PubMed: 17267749]

Herry C, Bach DR, Esposito F, Di Salle F, Perrig WJ, Scheffler K, Luthi A, Seifritz E. Processing of temporal unpredictability in human and animal amygdala. J Neurosci. 2007; 27:5958-5966. [PubMed: 17537966]

Huang YY, Kandel ER. Postsynaptic induction and PKA-dependent expression of LTP in the lateral amygdala. Neuron. 1998; 21:169-178. [PubMed: 9697861]

Huang YY, Kandel ER. Low-frequency stimulation induces a pathway-specific late phase of LTP in the amygdala that is mediated by PKA and dependent on protein synthesis. Learn Mem. 2007; 14:497-503. [PubMed: 17626908] 
Humeau Y, Herry C, Kemp N, Shaban H, Fourcaudot E, Bissiere S, Luthi A. Dendritic spine heterogeneity determines afferent-specific Hebbian plasticity in the amygdala. Neuron. 2005; 45:119-131. [PubMed: 15629707]

Humeau Y, Shaban H, Bissiere S, Luthi A. Presynaptic induction of heterosynaptic associative plasticity in the mammalian brain. Nature. 2003; 426:841-845. [PubMed: 14685239]

Kleschevnikov AM, Sokolov MV, Kuhnt U, Dawe GS, Stephenson JD, Voronin LL. Changes in paired-pulse facilitation correlate with induction of long-term potentiation in area CA1 of rat hippocampal slices. Neuroscience. 1997; 76:829-843. [PubMed: 9135055]

LeDoux JE. Emotion circuits in the brain. Annu Rev Neurosci. 2000; 23:155-184. [PubMed: 10845062]

Leung, LWS. Field potentials in the central nervous system: Recording, analysis, and modeling. In: Boulton, AA.; Baker, GB.; Vanderwolf, CH., editors. Neuromethods. Humana Press; Clifton, NJ: 1990.

Li XF, Armony JL, LeDoux JE. GABAA and GABAB receptors differentially regulate synaptic transmission in the auditory thalamo-amygdala pathway: an in vivo microiontophoretic study and a model. Synapse. 1996; 24:115-124. [PubMed: 8890453]

Likhtik E, Pelletier JG, Popescu AT, Pare D. Identification of basolateral amygdala projection cells and interneurons using extracellular recordings. J Neurophysiol. 2006; 96:3257-3265. [PubMed: 17110739]

Lindquist DH, Jarrard LE, Brown TH. Perirhinal cortex supports delay fear conditioning to rat ultrasonic social signals. J Neurosci. 2004; 24:3610-3617. [PubMed: 15071109]

Linke R, Braune G, Schwegler H. Differential projection of the posterior paralaminar thalamic nuclei to the amygdaloid complex in the rat. Exp Brain Res. 2000; 134:520-532. [PubMed: 11081834]

Marder CP, Buonomano DV. Differential effects of short- and long-term potentiation on cell firing in the CA1 region of the hippocampus. J Neurosci. 2003; 23:112-121. [PubMed: 12514207]

Maren S, Quirk GJ. Neuronal signalling of fear memory. Nat Rev Neurosci. 2004; 5:844-852. [PubMed: 15496862]

Martin SJ, Grimwood PD, Morris RG. Synaptic plasticity and memory: an evaluation of the hypothesis. Annu Rev Neurosci. 2000; 23:649-711. [PubMed: 10845078]

Mascagni F, McDonald AJ, Coleman JR. Corticoamygdaloid and corticocortical projections of the rat temporal cortex: a Phaseolus vulgaris leucoagglutinin study. Neuroscience. 1993; 57:697-715. [PubMed: 8309532]

McDonald AJ. Cortical pathways to the mammalian amygdala. Prog Neurobiol. 1998; 55:257-332. [PubMed: 9643556]

McKernan MG, Shinnick-Gallagher P. Fear conditioning induces a lasting potentiation of synaptic currents in vitro. Nature. 1997; 390:607-611. [PubMed: 9403689]

Mitzdorf U. Current source-density method and application in cat cerebral cortex: investigation of evoked potentials and EEG phenomena. Physiol Rev. 1985; 65:37-100. [PubMed: 3880898]

Nathan T, Lambert JD. Depression of the fast IPSP underlies paired-pulse facilitation in area CA1 of the rat hippocampus. J Neurophysiol. 1991; 66:1704-1715. [PubMed: 1684990]

Rodrigues SM, Schafe GE, LeDoux JE. Molecular mechanisms underlying emotional learning and memory in the lateral amygdala. Neuron. 2004; 44:75-91. [PubMed: 15450161]

Rogan MT, Staubli UV, LeDoux JE. Fear conditioning induces associative long-term potentiation in the amygdala. Nature. 1997; 390:604-607. [PubMed: 9403688]

Romanski LM, LeDoux JE. Equipotentiality of thalamo-amygdala and thalamo-cortico-amygdala circuits in auditory fear conditioning. J Neurosci. 1992; 12:4501-4509. [PubMed: 1331362]

Romanski LM, LeDoux JE. Organization of rodent auditory cortex: anterograde transport of PHA-L from MGv to temporal neocortex. Cereb Cortex. 1993; 3:499-514. [PubMed: 7511011]

Rosenkranz JA, Grace AA. Dopamine attenuates prefrontal cortical suppression of sensory inputs to the basolateral amygdala of rats. J Neurosci. 2001; 21:4090-4103. [PubMed: 11356897]

Schroeder BW, Shinnick-Gallagher P. Fear learning induces persistent facilitation of amygdala synaptic transmission. Eur J Neurosci. 2005; 22:1775-1783. [PubMed: 16197518] 
Shi CJ, Cassell MD. Cortical, thalamic, and amygdaloid projections of rat temporal cortex. J Comp Neurol. 1997; 382:153-175. [PubMed: 9183686]

Shin RM, Tsvetkov E, Bolshakov VY. Spatiotemporal asymmetry of associative synaptic plasticity in fear conditioning pathways. Neuron. 2006; 52:883-896. [PubMed: 17145508]

Sigurdsson T, Doyere V, Cain CK, LeDoux JE. Long-term potentiation in the amygdala: a cellular mechanism of fear learning and memory. Neuropharmacology. 2007; 52:215-227. [PubMed: 16919687]

Sokal D, Giarola A, Large C. Effects of GABA(B), 5-HT(1A), and 5-HT(2) receptor stimulation on activation and inhibition of the rat lateral amygdala following medial geniculate nucleus stimulation in vivo. Brain Res. 2005; 1031:141-150. [PubMed: 15621024]

Son $\mathrm{H}$, Carpenter DO. Interactions among paired-pulse facilitation and post-tetanic and long-term potentiation in the mossy fiber-CA3 pathway in rat hippocampus. Synapse. 1996; 23:302-311. [PubMed: 8855515]

Szinyei C, Heinbockel T, Montagne J, Pape HC. Putative cortical and thalamic inputs elicit convergent excitation in a population of GABAergic interneurons of the lateral amygdala. J Neurosci. 2000; 20:8909-8915. [PubMed: 11102501]

Tsvetkov E, Carlezon WA, Benes FM, Kandel ER, Bolshakov VY. Fear conditioning occludes LTPinduced presynaptic enhancement of synaptic transmission in the cortical pathway to the lateral amygdala. Neuron. 2002; 34:289-300. [PubMed: 11970870]

Turner BH, Herkenham M. Thalamoamygdaloid projections in the rat: a test of the amygdala's role in sensory processing. J Comp Neurol. 1991; 313:295-325. [PubMed: 1765584]

Volgushev M, Voronin LL, Chistiakova M, Singer W. Relations between long-term synaptic modifications and paired-pulse interactions in the rat neocortex. Eur J Neurosci. 1997; 9:16561665. [PubMed: 9283820]

Weisskopf MG, Bauer EP, LeDoux JE. L-type voltage-gated calcium channels mediate NMDAindependent associative long-term potentiation at thalamic input synapses to the amygdala. $\mathrm{J}$ Neurosci. 1999; 19:10512-10519. [PubMed: 10575047]

Wigstrom H, Gustafsson B. Facilitated induction of hippocampal long-lasting potentiation during blockade of inhibition. Nature. 1983a; 301:603-604. [PubMed: 6298626]

Wigstrom H, Gustafsson B. Large long-lasting potentiation in the dentate gyrus in vitro during blockade of inhibition. Brain Res. 1983b; 275:153-158. [PubMed: 6313124]

Wigstrom H, Gustafsson B. A possible correlate of the postsynaptic condition for long-lasting potentiation in the guinea pig hippocampus in vitro. Neurosci Lett. 1984; 44:327-332. [PubMed: 6145123]

Yaniv D, Schafe GE, LeDoux JE, Richter-Levin G. Perirhinal cortex and thalamic stimulation induces LTP in different areas of the amygdala. Ann N Y Acad Sci. 2000; 911:474-476. [PubMed: 10911897]

Zinebi F, Mckernan M, Shinnick-Gallagher P. Expression of fear-conditioning is accompanied by increased paired-pulse depression within the amygdala. Pharmacology Biochemistry and Behavior. 2002; 71:393-400.

Zinebi F, Russell R, McKernan M, Shinnick-Gallagher P. Comparison of paired-pulse facilitation of AMPA and NMDA synaptic currents in the lateral amygdala. Synapse. 2001; 42:115-127. [PubMed: 11574948]

Zucker RS, Regehr WG. Short-term synaptic plasticity. Annu Rev Physiol. 2002; 64:355-405. [PubMed: 11826273] 
A

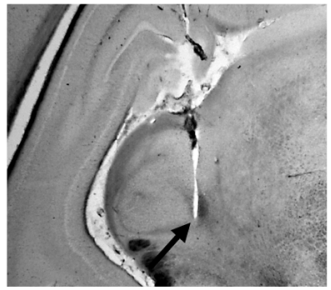

MGm/PIN

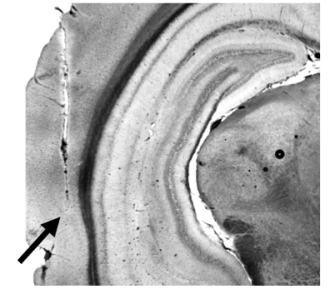

TE3

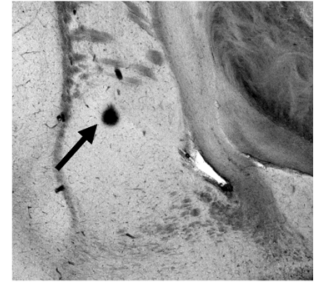

LA

B

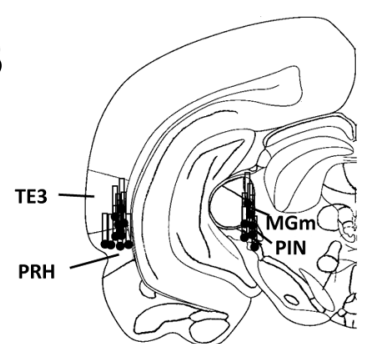

Bregma

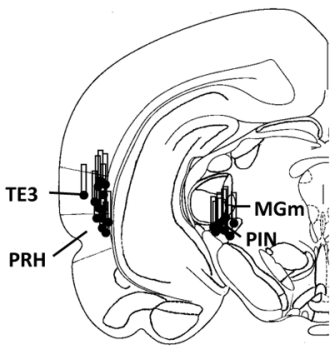

$-5.6$

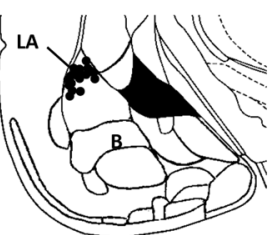

$-3.60$

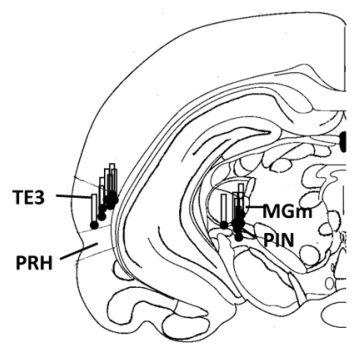

$-5.3$

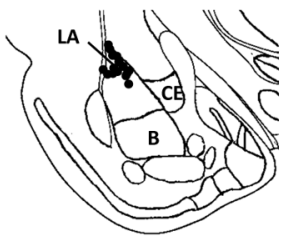

$-3.30$

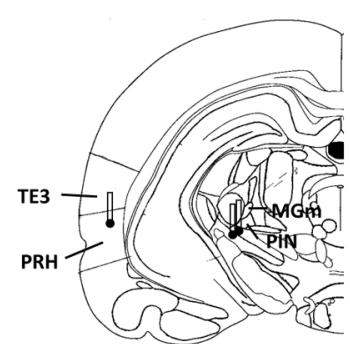

$-4.8$

C

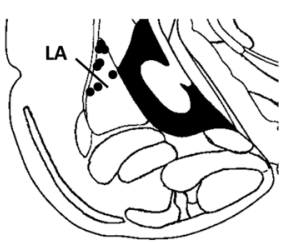

Bregma

$-3.80$

Figure 1. Electrode placements

$A$, Placements of stimulating electrodes in auditory association cortex (TE3) and auditory thalamus (MGm/PIN). Filled circles represent the electrode tips and the bars represent the distance between the tip and base of the bipolar stimulating electrode $(1 \mathrm{~mm})$ and thus the area of stimulation along the dorsoventral axis. $B$, Recording electrode placements in the LA. Because of overlap, not all individual recording sites are visible. 
A

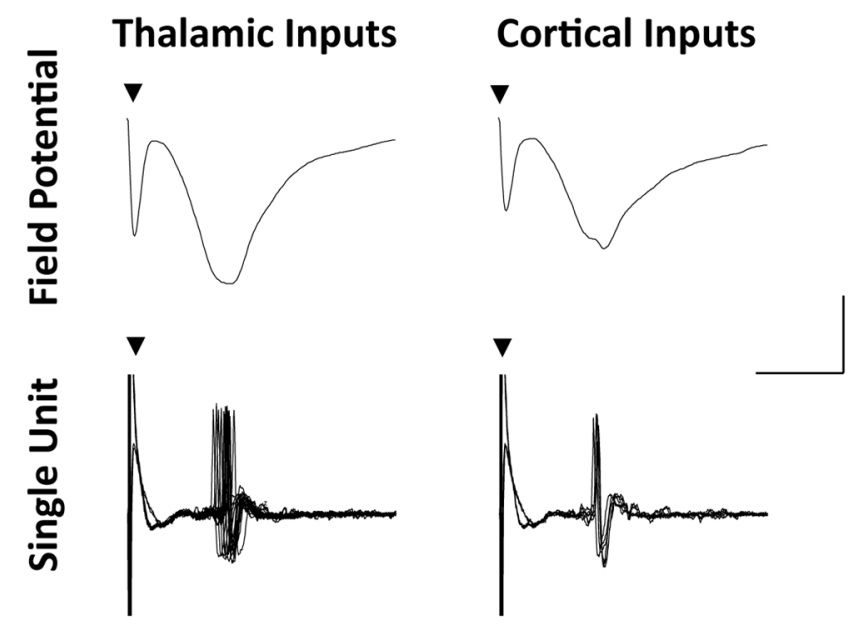

B
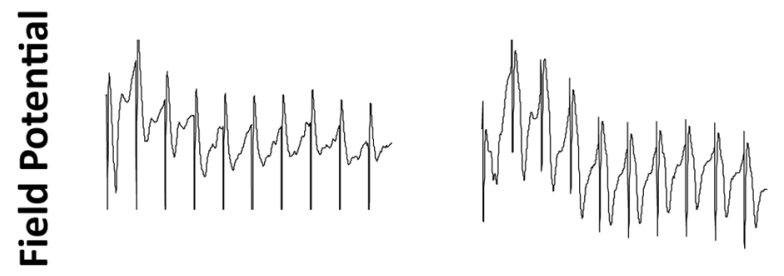

C

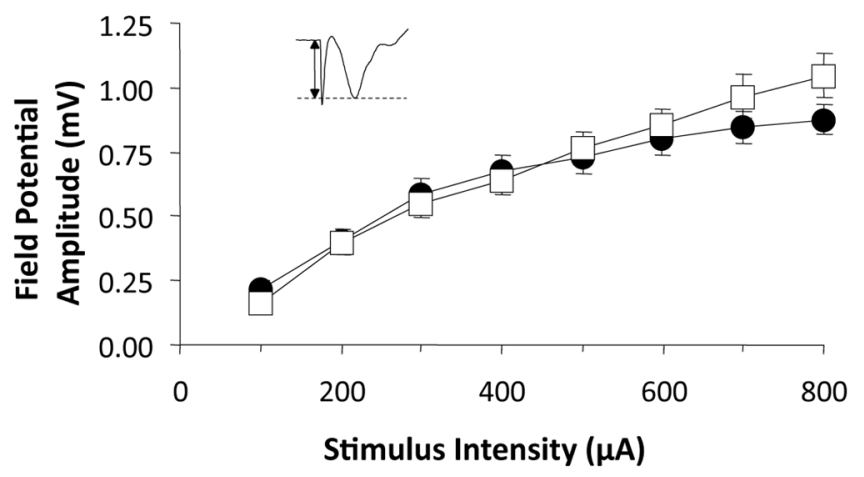

- Thalamic Inputs $\quad \square-$ Cortical Inputs

Figure 2. Response characteristics of thalamic and cortical inputs in vivo

$A$, Representative examples of neural responses recorded in the LA following stimulation of the thalamic or cortical pathway, respectively, in the same animal. Upper traces show field potential responses, whereas lower traces show the response of a single neuron recorded from the same electrode which was responsive to stimulation of both pathways (10 sweeps). Scale bar $0.5 \mathrm{mV}$ (field potentials), $1.0 \mathrm{mV}$ (single-unit), $5 \mathrm{~ms}$. Triangles indicate stimulus artifacts. Note the temporal correspondence between field potential and single-unit responses. $B$, Mean $( \pm$ SEM) amplitude of field potentials as a function of stimulus intensity applied to the two pathways $(n=16)$. Amplitude is defined by figure in inset. 
A

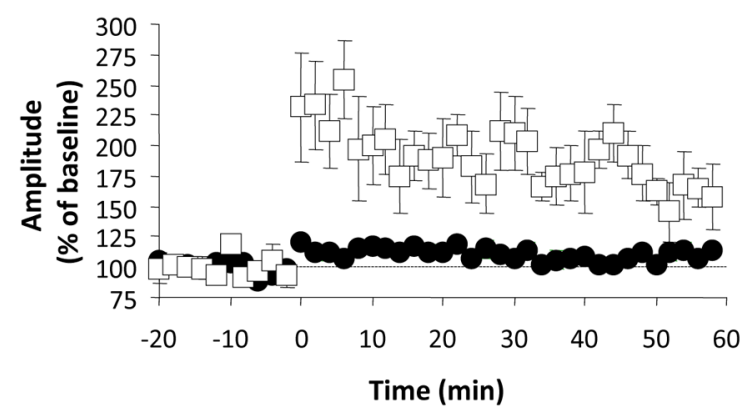

- Thalamic Inputs $\square$ Cortical Inputs

\section{B}

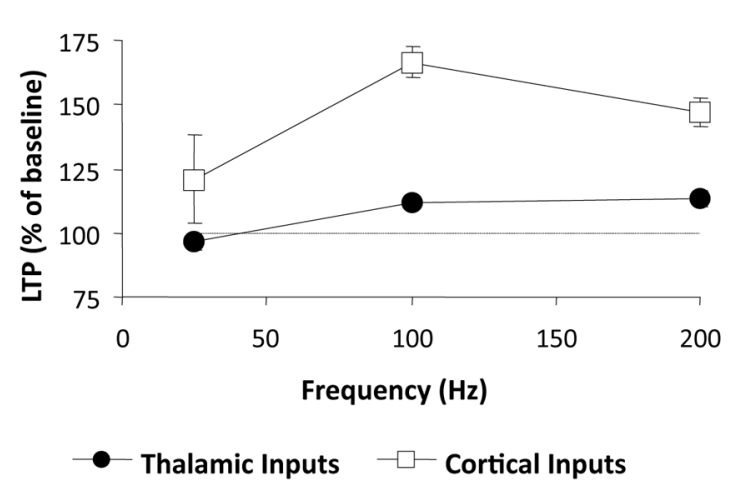

C

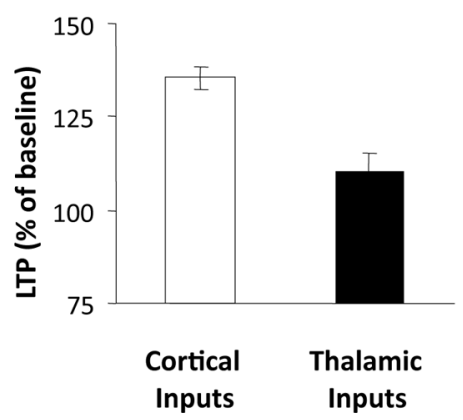

Figure 3. Long-term potentiation at thalamic and cortical inputs

$A$, LTP induced by 3 series of $100 \mathrm{~Hz}$ trains at the thalamic ( $n=5)$ and cortical $(n=5)$ inputs. Values represent mean $( \pm$ SEM) amplitudes expressed as \% of baseline values. Traces on the right show representative responses before and after LTP induction. Scale bar: $1 \mathrm{mV}, 5 \mathrm{~ms}$. $B$, LTP as a function of HFS frequency. Values represent LTP (mean \pm SEM), measured 1 $\mathrm{hr}$ after induction following $25 \mathrm{~Hz}$ ( $n=4$ and 3), $100 \mathrm{~Hz}(n=5$ and 5) and $200 \mathrm{~Hz}(n=4$ and 3) stimulation ( $n$ values represent thalamic and cortical inputs, respectively). $C$, LTP following theta-burst stimulation ( $\mathrm{n}=5$ per input). 
A

\section{$100 \mathrm{~Hz}$}
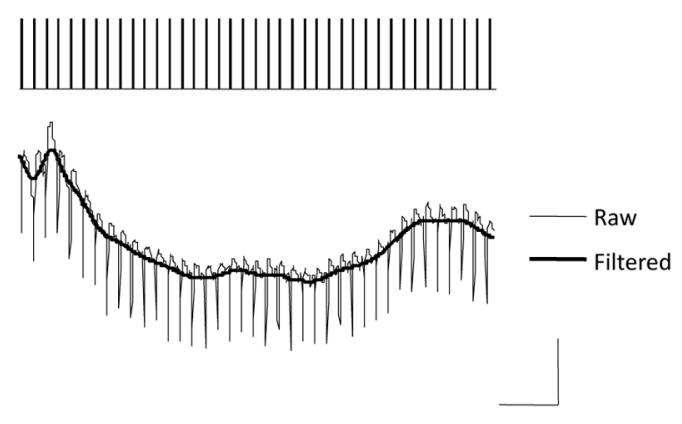

C

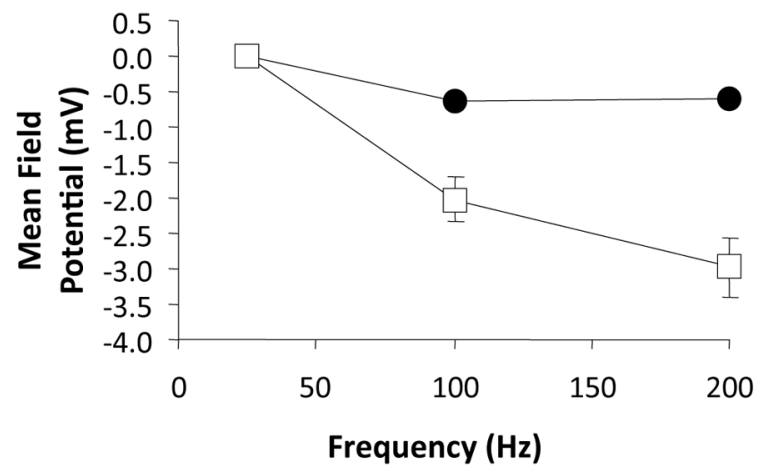

B

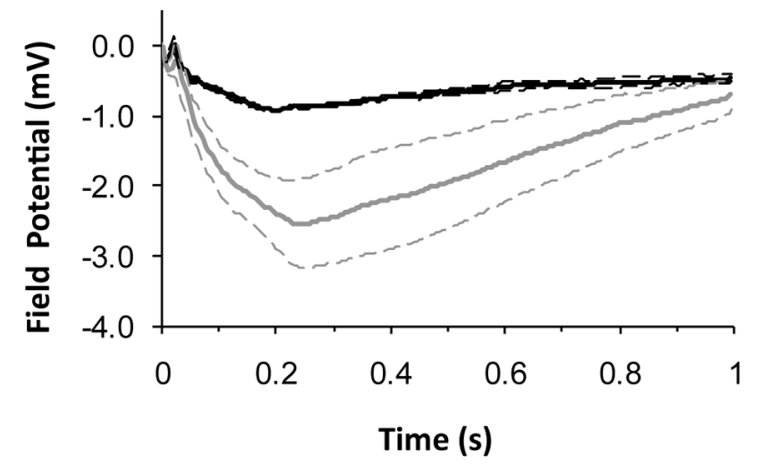

Thalamic Input $\quad$ Cortical Input

D

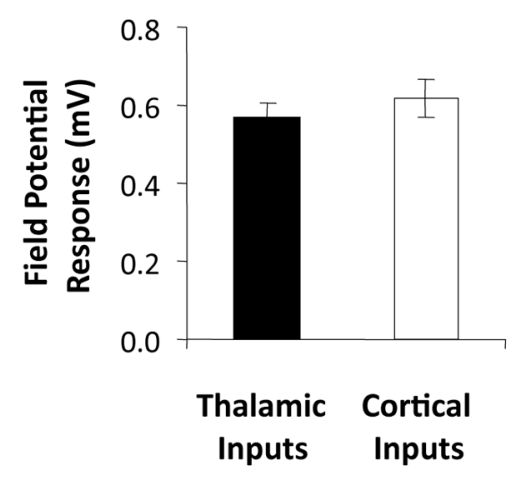

\section{- Thalamic Inputs $\quad \square-$ Cortical Inputs}

Figure 4. Responses during high-frequency stimulation of thalamic and cortical inputs $A$, Initial segment of a response recorded in the LA during HFS at $100 \mathrm{~Hz}$. Traces were filtered digitally as shown to remove stimulus artifacts prior to further analysis. Scale bar: 1 $\mathrm{mV}, 50 \mathrm{~ms}$. B, Mean ( \pm SEM) responses during $100 \mathrm{~Hz}$ HFS. Values represent the field potential relative to the pre-HFS baseline. $C$, Field potential (mean \pm SEM) during HFS as a function of frequency ( $n$ values as in Figure 3 ). Values represent the mean field potential during HFS. $D$, Mean $( \pm$ SEM) test stimulus responses prior to HFS $(n=15$ and 13 for thalamic and cortical inputs, respectively). 


\section{A}

B
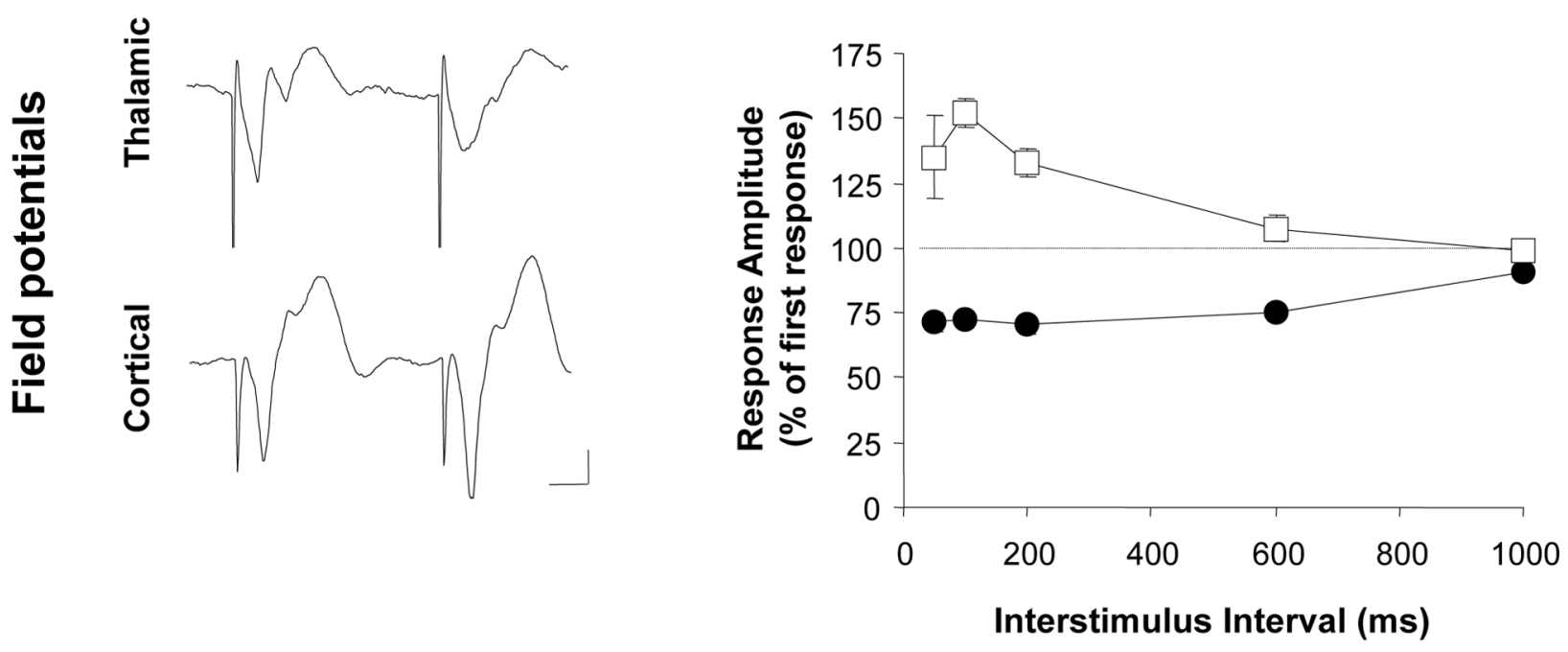

C

D
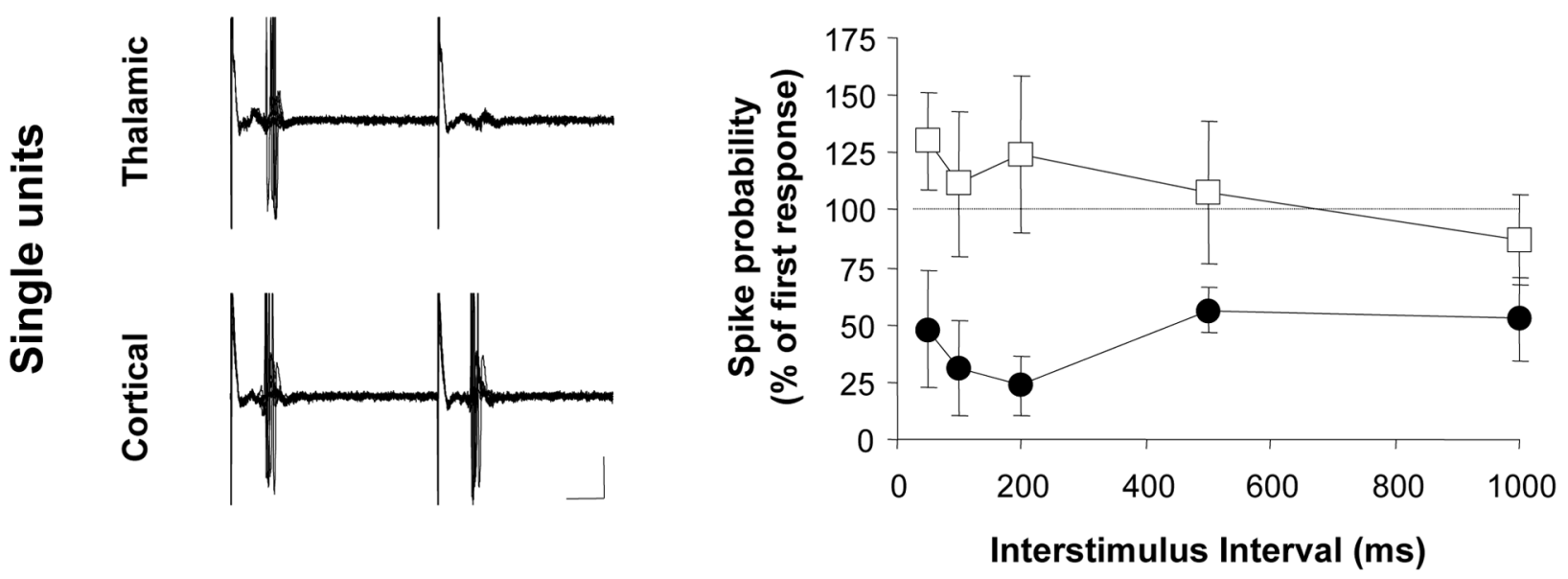

- Thalamic Inputs $-\square-$ Cortical Inputs

Figure 5. Divergent paired-pulse response profiles at thalamic and cortical inputs $A$, Representative field potential responses during paired-pulse stimulation with a $50 \mathrm{~ms}$ inter-stimulus interval (ISI). Scale bar: $0.25 \mathrm{mV}, 10 \mathrm{~ms}$. $B$, Mean ( \pm SEM) amplitudes of the responses to the second pulse in a pair (expressed as \% of the response to the first pulse) in all animals tested $(n=17)$. $C$, Representative example of a single unit responding to both thalamic and cortical paired-pulse stimulation at $50 \mathrm{~ms}$ ISI. Scale bar: $200 \mu \mathrm{V}, 10 \mathrm{~ms} . D$, Mean ( \pm SEM) probability of eliciting a spike with the second stimulus in a pair (expressed as $\%$ of the probability associated with the first stimulus) for all neurons tested $(n=9)$. 


\section{Thalamic inputs}

A

Saline

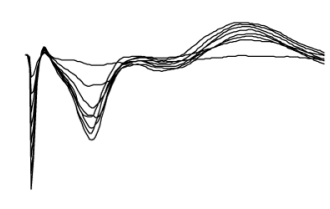

C

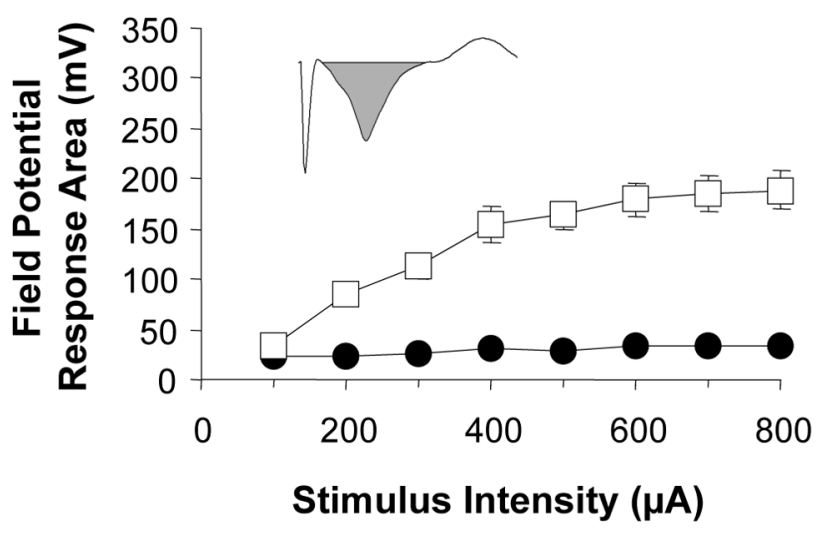

- Saline $-\square-$ Picrotoxin

\section{Cortical inputs}

B

Saline

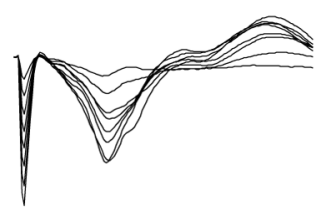

D

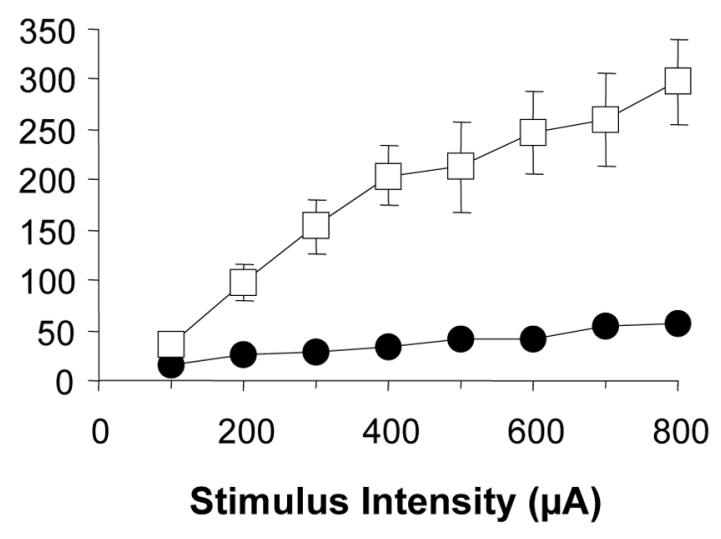

$\rightarrow$ Saline $-\square-$ Picrotoxin

Figure 6. Effect of $\mathrm{GABA}_{\mathbf{A}}$ blockade on evoked responses in the LA

$A, B$, Representative responses recorded in the LA in response to stimulation of thalamic and cortical inputs, respectively, at increasing intensities with either picrotoxin or saline in the recording pipette. Scale bar: $1 \mathrm{mV}, 20 \mathrm{~ms}$. $C, D$, Mean $( \pm \mathrm{SEM})$ response area (see inset) as a function of stimulus intensity with either picrotoxin or saline in the recording pipette ( $n=12$ and 16 animals for recordings with picrotoxin and saline, respectively). 


\section{Thalamic inputs}

A

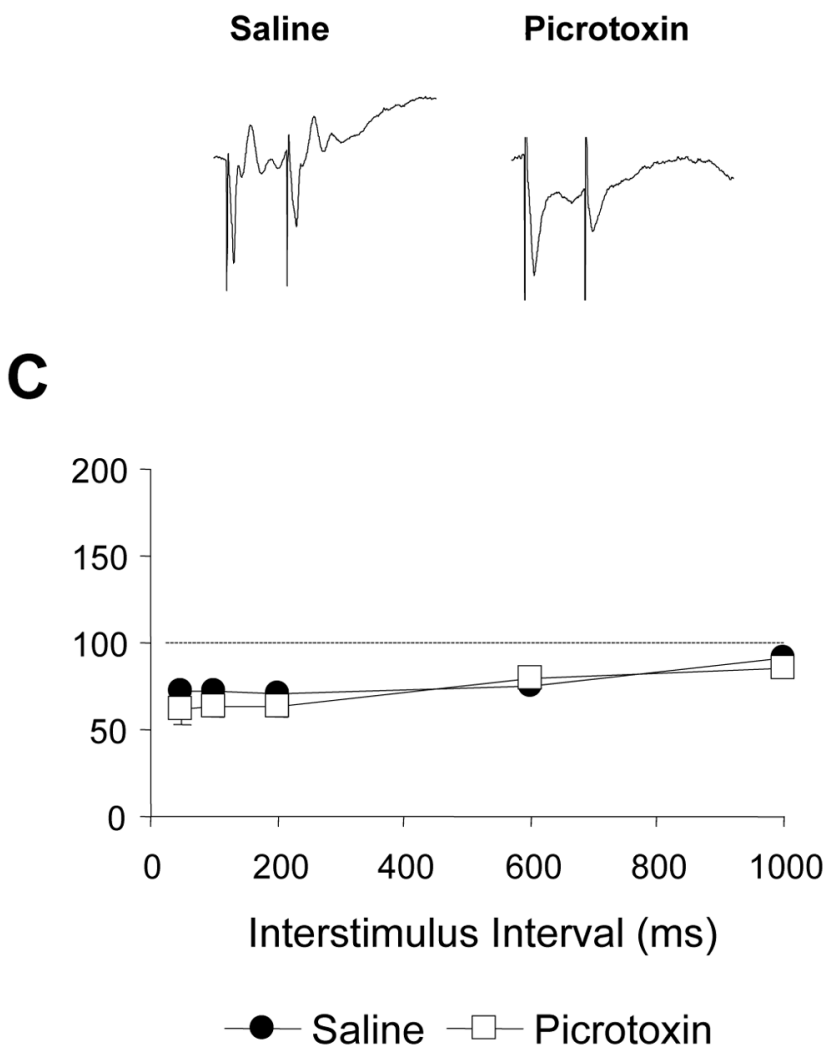

Cortical inputs

B
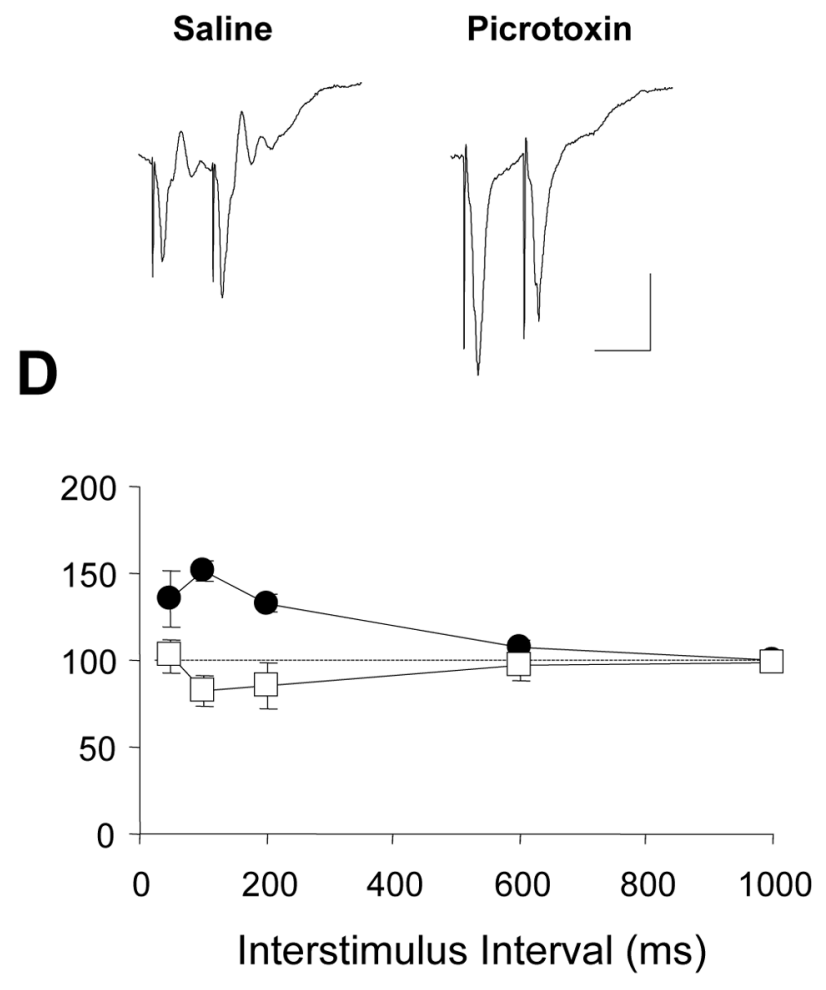

- Saline $-\square$-Picrotoxin

Figure 7. Effect of $\mathrm{GABA}_{\mathbf{A}}$ blockade on paired-pulse responses $A, B$, examples of paired-pulse responses (50ms ISI) at thalamic and cortical inputs, respectively, recorded in the presence of saline or picrotoxin. Scale bar: $0.5 \mathrm{mV}, 50 \mathrm{~ms}$. $C, D$, Mean $( \pm \mathrm{SEM})$ paired-pulse responses at the two inputs in the presence of either saline $(\mathrm{n}=16)$ or picrotoxin $(\mathrm{n}=12)$. 
Thalamic inputs

A

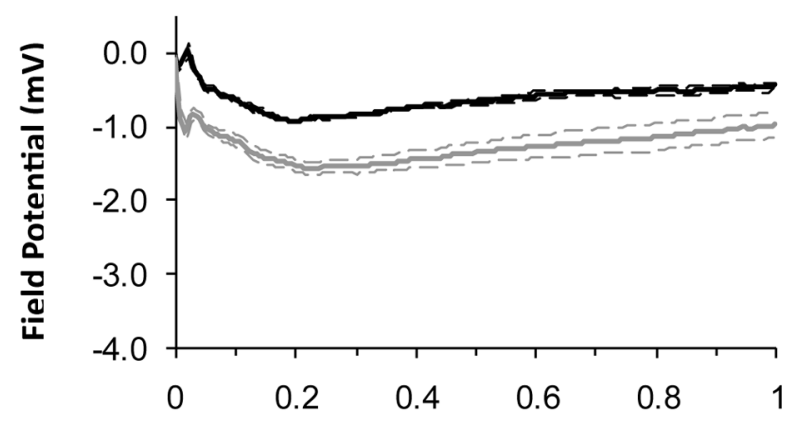

Saline $=$ Picrotoxin

C

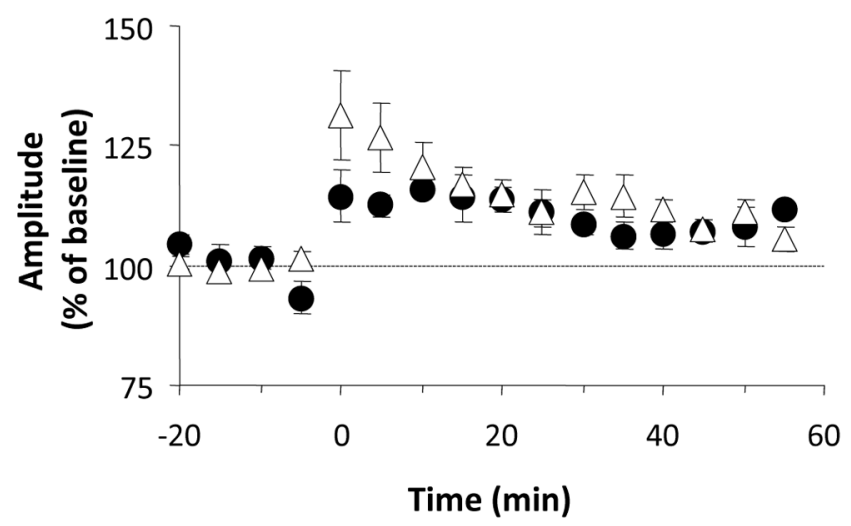

- Saline $\triangle$ Picrotoxin
B

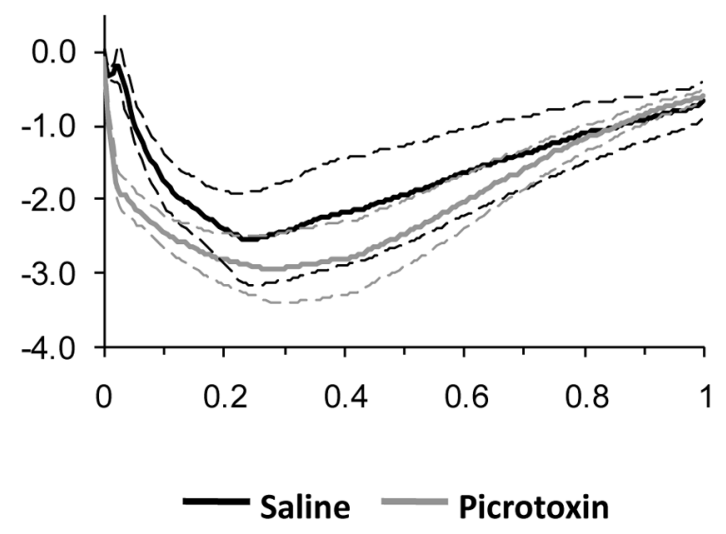

D

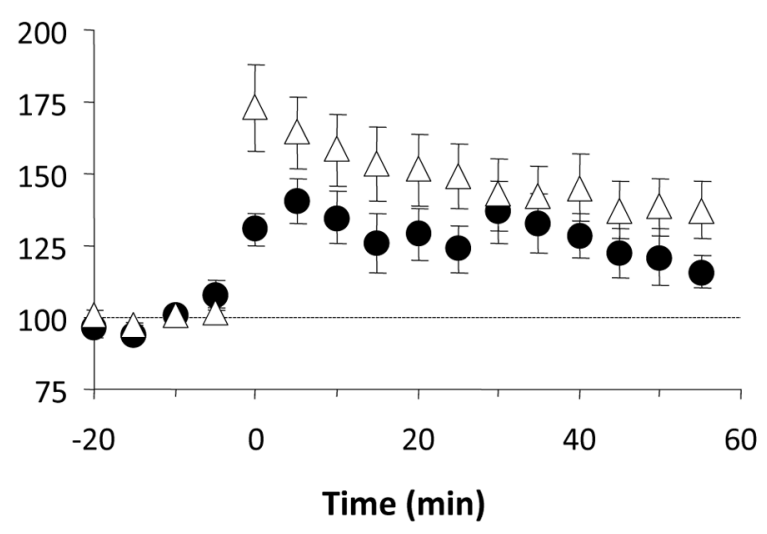

Saline $\triangle$ Picrotoxin

Figure 8. Effect of GABA $\mathbf{A}$ blockade on LTP induction in the LA

Responses to $100 \mathrm{~Hz} \operatorname{HFS}(A, B)$ and $\operatorname{LTP}(C, D)$ at thalamic and cortical inputs, respectively, with either saline ( $\mathrm{n}=5$ per input) or picrotoxin $(\mathrm{n}=9-11$ per input) in the recording pipette. Values represent mean \pm SEM. 